\title{
Metamorphic III-V solar cells: recent progress and potential
}

\author{
Iván Garcia , Ryan M. France , John F. Geisz, \\ William E. McMahon, Myles A. Steiner, Daniel J. Friedman
}

\begin{abstract}
Metamorphic semiconductor devices are commonly considered to have inferior electronic quality. However, recent development of compositionally graded buffers and junction structures have led to the achievement of high quality metamorphic solar cells exhibiting internal luminescence efficiencies over $90 \%$. Optimizing the optical design of the solar cell becomes important in order to enhance photon recycling and open circuit voltage in these cells. In this paper we first present recent performance results for $1 \mathrm{eV}$ and $0.7 \mathrm{eV}$ GaInAs solar cells grown on GaAs substrates. Then an electro-optical model is used to assess the potential performance improvements in current metamorphic solar cells under different realizable design scenarios. The results show that significant improvements can be achieved by improving both the electronic quality and optics of these cells.
\end{abstract}

\section{INTRODUCTION}

The metamorphic growth approach pursues expanding the range of bandgaps achievable in multijunction solar cells by using different lattice constants than the substrate. The target lattice constant is accessed by slowly relieving the strain during the growth of compositionally graded buffers, which consist of epitaxial layers with increasing lattice constant. This strain relief is achieved by the controlled introduction of lattice dislocations. Residual threading dislocations (TD) are the main cause of the inferiority generally attributed to latticemismatched semiconductor structures, in terms of electronic quality. The higher the threading dislocation density (TDD), the worse the performance of the solar cell. A TDD of $\sim 1 \bullet 10^{5}$ is usually taken as the figure below which the performance of solar cell with typical thickness of $\sim 3 \mu \mathrm{m}$ is not limited by the TD [1]. This residual TDD is normally difficult to achieve, and TDDs in the range of $1 \cdot 10^{6}$ to $1 \cdot 10^{7}$ are typical in solar cell applications.

In this paper we present our recent progress on metamorphic junctions for 4-junction solar cells. We show high quality $1 \mathrm{eV}$ and $0.7 \mathrm{eV}$ metamorphic solar cells with internal radiative efficiencies $>90 \%$ and $>50 \%$, respectively. Then, we assess the performance potential of these cells and identify pathways for future improvements.

\section{RECENT PROGRESS ON METAMORPHIC JUNCTIONS}

Metamorphic compositionally graded buffers (CGB) are used to access the desired lattice constant. The residual TDD at the end of a CGB is minimized if the nucleation of dislocations is kept at the minimum necessary to start and sustain relaxation by dislocation glide throughout the grade growth. A kinetic model exists that describes the effect of grow th conditions and CGB structure on dislocation glide and the expected residual TDD in an ideal CGB [2]. However, in practice, many factors are at play that hinder the glide of TD and force the nucleation of new dislocations, giving rise to higher residual TDD. Among these factors are imperfections in the material such as impurities, roughness, compositional fluctuations, etc.

Single domain ordering in GaInP was recently found to enhance the glide and modify the plane distribution of TD in GaInP-based CGBs [3], [4]. Dislocations gliding across the ordered planes can disrupt the ordering pattern by the formation of antiphase domains in $\mathrm{B}+$ planes [3]. Since disordered GaInP has a lower energy than ordered GaInP, the formation of these antiphase boundaries releases energy and acts as an additional driving force for dislocation motion through the ordered planes.

This effect has been taken advantage of to achieve high quality GaInP-based CGBs for GaInAs subcells in 3-junction and 4-junction solar cells. We presented $1 \mathrm{eV} \mathrm{Ga}{ }_{0.73} \mathrm{In}_{0.27} \mathrm{As}$ solar cells grown on GaAs substrates (lattice misfit $\sim 2 \%$ ) with TDD $2 \cdot 10^{6} \mathrm{~cm}^{-2}$ using thin CGBs [5]. In this work we present new advances in this solar cell. We show TDD $\sim 5 \cdot 10^{5} \mathrm{~cm}^{-2}$ achieved by enhancing the dislocation glide through optimization of the growth conditions in the CGB with the glide enhancement from atomic ordering in mind. For this purpose, a varying growth temperature is used aiming to minimize phase separation and enhance the ordering in each step of the CGB. In figure 1 we show a reciprocal space map of one of these solar cells for the (004) reflection from (110) incidence. The epilayer tilt increases throughout the CGB due to the glide enhancement from atomic ordering. The inset EBIC image was measured on this cell and illustrates the very low TDD achieved (a TDD $\sim 5 \bullet 10^{5} \mathrm{~cm}^{-2}$ was obtained as the average of the TDD in multiple EBIC images).

The carrier collection efficiency and external quantum efficiency (EQE) of these solar cells is near perfect. However, the carrier collection efficiency is less sensitive to the TDD than other performance parameters, and previous $1 \mathrm{eV}$ cell designs already exhibited very good external quantum efficiencies [5]. In the new design presented here, the open circuit voltage $\left(\mathrm{V}_{o c}\right)$ and the internal and external luminescence efficiencies $\left(\eta_{\text {int }}\right.$ and $\left.\eta_{\text {ext }}\right)$ are the exceling parameters. Both $\eta_{\text {int }}$ and $\eta_{\text {ext }}$ are useful for the assessment of 


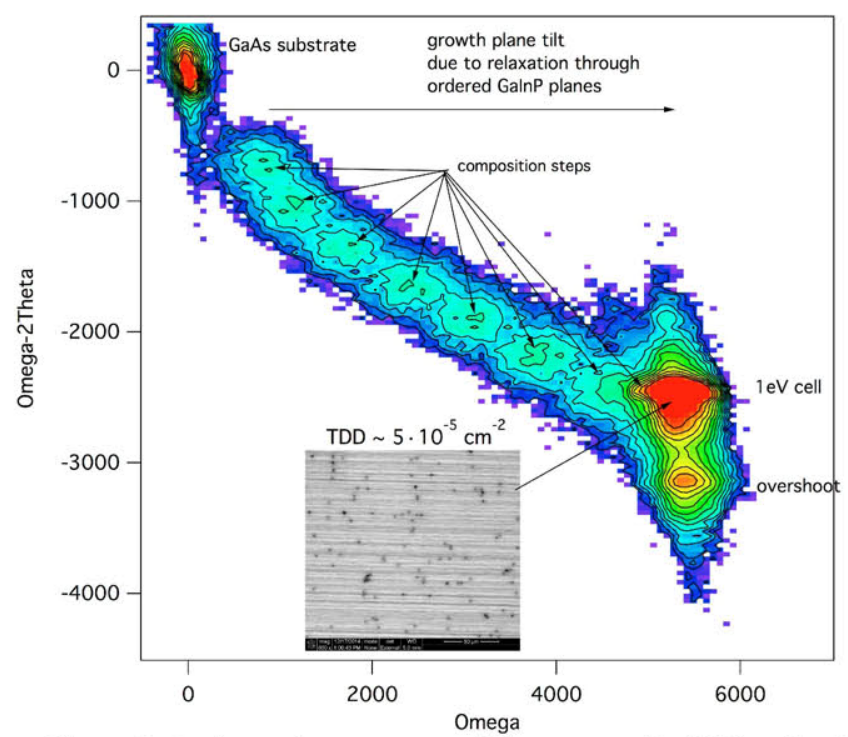

Figure 1. Reciprocal space map of the symmetric (004) reflection of a $1 \mathrm{eV}$ GaInAs solar cell grown on a GaAs substrate. The inset is an EBIC image of this cell, showing the threading dislocations.

the solar cell performance because they are directly linked to the electro-optical quality and do not depend directly on the bandgap: $\eta_{\text {int }}$ is a direct measure of the electronic quality of the junction while $\eta_{\text {ext }}$ represents both the electronic quality and the ability of the optical design to extract the emitted light.

In Figure 2 the $\eta_{\text {int }}$ and $\eta_{\text {ext }}$, obtained through electroluminescence measurements and optical modeling [6] (details in final manuscript) is shown for these $1 \mathrm{eV}$ cells, as well as for $0.7 \mathrm{eV}$ cells explained next. For the $1 \mathrm{eV}$ cells, the $\eta_{\text {int }}$ reaches a value of 0.9 at the highest current density used (limited by the measurement instruments). This can be compared to the highest $\eta_{\text {int }}$ achieved in lattice-matched GaAs, ( $0.98[6])$. At this high $\eta_{\text {int }}$, photon recycling effects start to be significant [7], and they can be taken advantage of to raise the performance of the solar cell, as shown in next section.

High quality $\sim 0.7 \mathrm{eV} \mathrm{Ga}_{0.5} \mathrm{In}_{0.5} \mathrm{As}$ solar cells grown on GaAs substrates (lattice misfit $\sim 4 \%$ ) have also been developed and are an essential part in the recent development of 4-junction solar cells [8], [9] which exhibit record photovoltaic conversion efficiencies [10]. GaInP-based CGBs are also used for this subcell. In addition to the large lattice mismatch with respect to the GaAs substrate, the development of these GaInP-based CGBs has to mitigate the nucleation of dislocations associated with the glide plane switch that occurs as the CGB composition changes towards InP, and dislocation energetics change [3], [11]. Despite these challenges, residual TDD as low as $\sim 2 \bullet 10^{6}$ have been achieved. The $\eta_{\text {int }}$ and $\eta_{\text {ext }}$ of one of these cells is shown in Figure 2, and can be observed to be significantly lower than in the case of the $1 \mathrm{eV}$ cells. However, a similar progression with the current density can be observed, and a $\eta_{\text {int }}$ of $>50 \%$ was obtained for the higher current density tested. It is possible that other factors related to

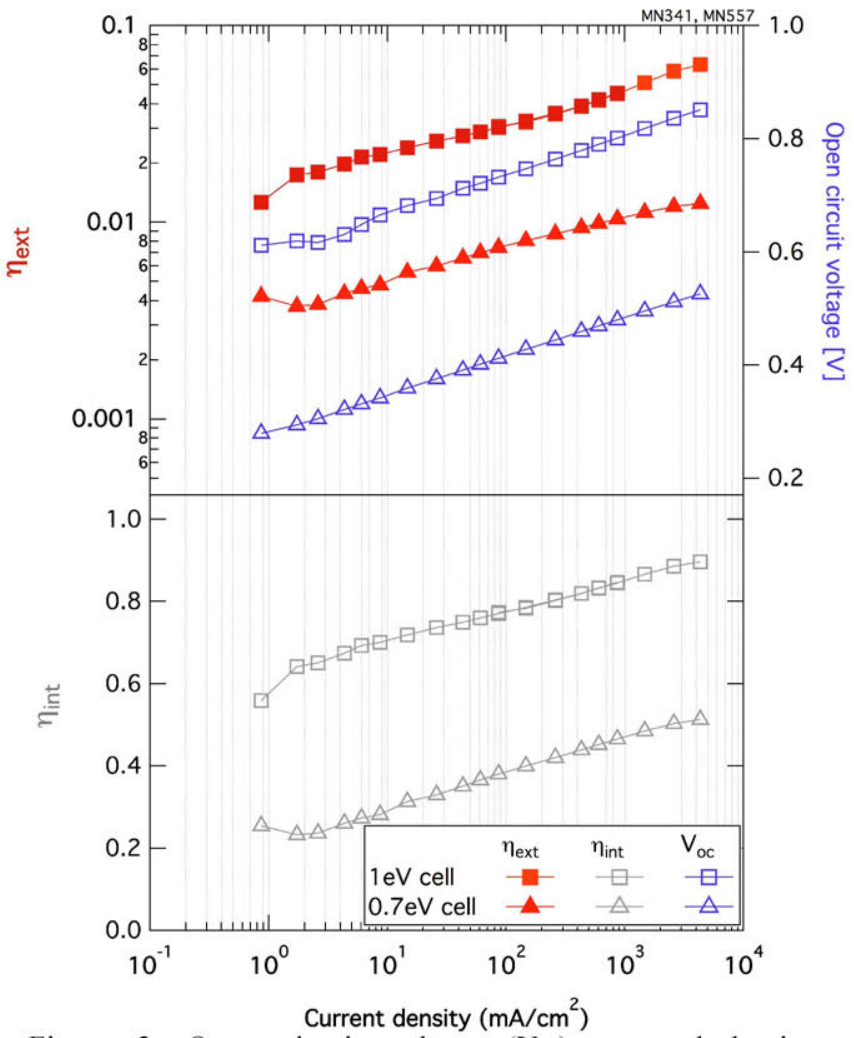

Figure 2. Open circuit voltage $\left(\mathrm{V}_{\mathrm{oc}}\right)$, external luminescence efficiency $\left(\eta_{\text {ext }}\right)$ and internal luminescence efficiency $\left(\eta_{\text {int }}\right)$ of metamorphic $1 \mathrm{eV}$ and $0.7 \mathrm{eV}$ cells grown on GaAs substrates.

lattice-mismatch may limit the performance of these subells, such as compositional variation or interfacial dislocations.

\section{POTENTIAL}

Using the same electro-optical model, the performance potential of these metamorphic solar cells was assessed. The focus was put on the $V_{o c}$, since the carrier collection efficiency is already very good. The $\mathrm{V}_{\mathrm{oc}}$ depends on the electronic properties of the solar cell. When the electronic quality is high enough, the optics of the solar cell semiconductor structure also plays an important role because they determine the photon recycling. The angle-integrated reflectance at the back of the solar cell is the most accessible knob in the optical structure to modify the photon recycling [6]. For singlejunction solar cells, it can be varied by modifying the properties of the back reflector and absorbing back layers in the semiconductor structure. In a multijunction solar cell, the ability to tweak the back reflectance depends on the possibility of placing a reflector structure at the back of the subcell. In figure $3 \mathrm{~V}_{\mathrm{oc}}$ contours of the $1 \mathrm{eV}$ and $0.7 \mathrm{eV}$ cells are plotted against the internal luminescence efficiency and the back angle-integrated reflectance. In these plots, the datapoint corresponding to current experimental cell results is shown as a filled square marker. 

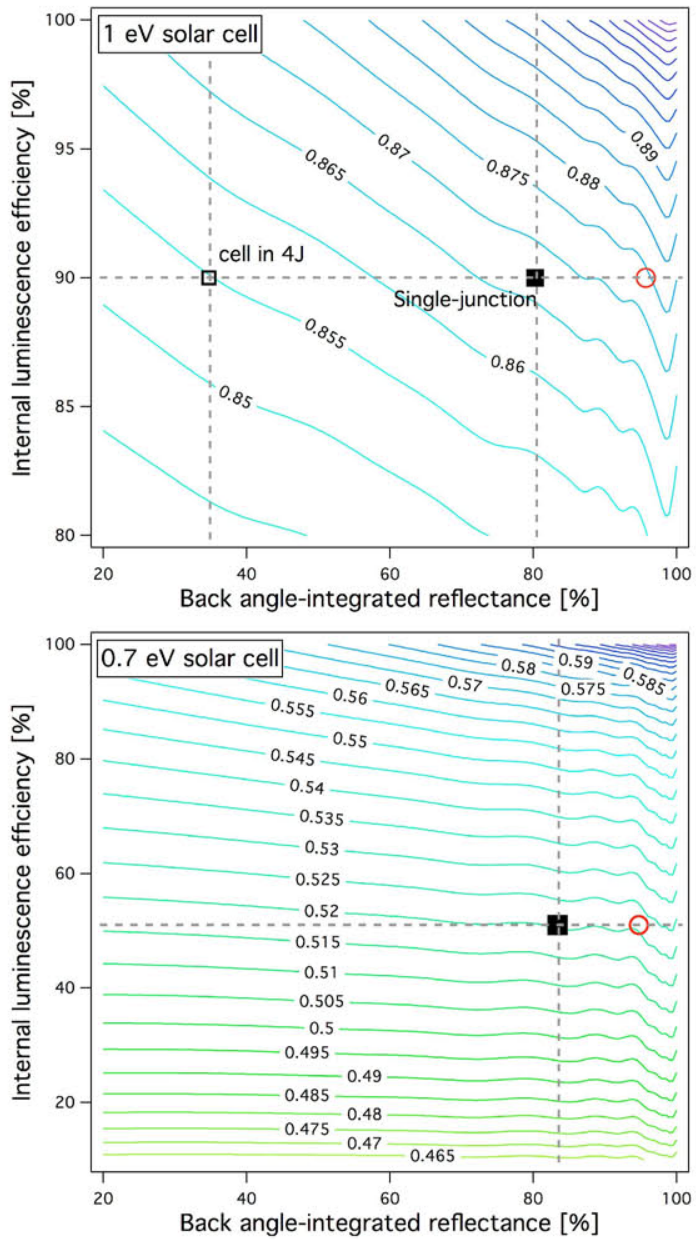

Figure $3 . \mathrm{V}_{\mathrm{oc}}$ of $1 \mathrm{eV}$ and $0.7 \mathrm{eV}$ metamorphic solar cells grown on GaAs substrates, plotted against the internal luminescence efficiency and the back angle-integrated reflectance. The filled square marker represents the experimental single-junction cells obtained so far. The open circular marker represents the design with low absorption at the back contact layer.

In the case of the $1 \mathrm{eV}$ cell, the material quality is high enough such that significant gains in $\mathrm{V}_{\mathrm{oc}}$ can be achieved if the back reflectance is increased. For example, the circular marker indicates the $\mathrm{V}_{\mathrm{oc}}$ that can be achieved when eliminating the absorption of luminescence in the back contact layer, for example by using a higher bandgap material such as AlGaInAs. The empty square symbol indicates the $\mathrm{V}_{\mathrm{oc}}$ of the device when placed in a 4-junction solar cell with a $0.7 \mathrm{eV}$ subcell below and no reflector in between. The voltage loss is significant, but placing a reflector in between both subcells to raise the $1 \mathrm{eV}$ subcell back reflectance can be a challenging task.

As for the $0.7 \mathrm{eV}$ subcell, the expected gains when improving the back reflectance, represented by the circular marker, are negligible as a consequence of the much lower internal luminescence efficiency. In this subcell the priority is increasing the internal luminescence efficiency.

\section{SUMMARY AND CONCLUSIONS}

High performance metamorphic junctions have been developed recently. We have shown $1 \mathrm{eV}$ cells with internal luminescence efficiencies over $90 \%$, which are high enough material quality to benefit from photon recycling. The electrooptical model used indicates significant $\mathrm{V}_{\text {oc }}$ gains in these cells by improving their optics. The internal luminescence efficiency of $0.7 \mathrm{eV}$ subcells is not high enough to benefit from photon recycling and the focus is put on the electronic quality for their development. Overall, these results show that very high quality metamorphic junctions can be achieved and further development of their electro-optical properties provides a realizable path for significant improvements in the conversion efficiency of 4-junction solar cells.

\section{REFERENCES}

[1] M. Yamaguchi and C. Amano, "Efficiency calculations of thin-film GaAs solar cells on Si substrates," J. Appl. Phys., vol. 58, no. 9, pp. 3601-3606, Nov. 1985.

[2] E. A. Fitzgerald, "Dislocations in strained-layer epitaxy: theory, experiment, and applications," Mater. Sci. Rep., vol. 7, no. 3, pp. 87-142, Nov. 1991.

[3] W. E. McMahon, J. Kang, R. M. France, A. G. Norman, D. J. Friedman, and S.-H. Wei, "Ordering-enhanced dislocation glide in III-V alloys," J. Appl. Phys., vol. 114, no. 20, p. 203506, Nov. 2013.

[4] R. M. France, W. E. McMahon, A. G. Norman, J. F. Geisz, and M. J. Romero, "Control of misfit dislocation glide plane distribution during strain relaxation of CuPt-ordered GaInAs and GaInP," J. Appl. Phys., vol. 112, no. 2, p. 023520, Jul. 2012.

[5] I. Garcia, R. M. France, J. F. Geisz, and J. Simon, "Thin, high quality GaInP compositionally graded buffer layers grown at high growth rates for metamorphic III-V solar cell applications," J. Cryst. Growth, vol. 393, pp. 64-69, May 2014

[6] M. A. Steiner, J. F. Geisz, I. García, D. J. Friedman, A. Duda, and S. R. Kurtz, "Optical enhancement of the open-circuit voltage in high quality GaAs solar cells," J. Appl. Phys., vol. 113, no. 12, p. 123109, Mar. 2013.

[7] O. D. Miller, E. Yablonovitch, and S. R. Kurtz, "Strong Internal and External Luminescence as Solar Cells Approach the Shockley-Queisser Limit," IEEE J. Photovolt., vol. 2, no. 3, pp. 303-311, Jul. 2012.

[8] R. M. France, I. Garcia, W. E. McMahon, A. G. Norman, J. Simon, J. F. Geisz, D. J. Friedman, and M. J. Romero, "LatticeMismatched $0.7-\mathrm{eV}$ GaInAs Solar Cells Grown on GaAs Using GaInP Compositionally Graded Buffers," IEEE J. Photovolt., vol. 4, no. 1, pp. 190-195, Jan. 2014.

[9] R. M. France, J. F. Geisz, I. Garcia, M. A. Steiner, W. E. McMahon, D. J. Friedman, T. E. Moriarty, C. Osterwald, J. Scott Ward, A. Duda, M. Young, and W. J. Olavarria, "Quadruple-Junction Inverted Metamorphic Concentrator Devices," IEEE J. Photovolt, vol. 5, no. 1, pp. 432-437, Jan. 2015.

[10] R. M. France, "Design flexibility of 4-junction inverted metamorphic solar cells," Submitt. This Conf.

[11] R. M. France, I. Garcia, W. E. McMahon, A. G. Norman, J. Simon, J. F. Geisz, D. J. Friedman, and M. J. Romero, "Lattice- 